\title{
THE EFFECT OF CONTROLLING THE WEIGHT VARIABLE ON THE TYPEFACE ATTRIBUTE ASSESSMENT
}

\author{
Jelena Žarko (D), Uroš Nedeljković \\ University of Novi Sad, Faculty of Technical Sciences, Department of Graphic Engineering and \\ Design, Novi Sad, Serbia
}

\begin{abstract}
Certain characteristics specific for typeface design initiate different impressions on observers, but here arises a research problem where we cannot identify what specific or universal characteristics of the typeface initiate the impressions on certain attributes. A common problem encountered in previous researches is that fonts may vary in many variables and at the same time differ in width, weight, contrast, and structure. Therefore, it is difficult to determine and isolate which universal and specific characteristics of the typeface affect the impression. The subject of this paper is the isolation of the weight variable and the investigation of its relationship with typeface personality attributes. The main goal of this paper is to provide empirical support for theoretical assumptions, building on previous researches on the typeface personality and typographic rhetoric to show how specific typographic characteristics influence the experience of writing itself.
\end{abstract}

Keywords:Typeface personality, Visual rhetoric, Universal structure

\section{INTRODUCTION}

According to Aristotle's theory of rhetoric, people are affected through reason (logos), through feelings (pathos) and personality, morals, ethics (ethos). Logos, Pathos and Ethos are key dimensions for communication efficiency. Rhetoric is a skill (ars techne), and skill is a set of rules that arose from experience and was subsequently thought out (Aristotele, et al., 1954). Typography as well as rhetoric insists on order, symmetry, limitations, structure and composition of parts as a whole. Beauty is the subject of both rhetoric and typography. The activities of typographers are similar to the activities of rhetoricians. Just like a rhetorician, a typographer applies rules of conduct to his art to ultimately lead his audience to see as he sees. Typography encompasses not only the visual impression of the typeface but also the relationship between the image we perceive and the statement we want to express. This harmony can only be successfully achieved through creative interpretation between rhetorician and typographer (Trummel, 1988).

Typeface design is an important visual tool for achieving the goals of communication in general, and today, in particular, technical communication. The design of the typeface affects the impressions of the observer, but the nature of that effect is still unknown. The personality of the typeface is an important aspect of technical documents and the education of administrative staff. By selecting the appropriate font, the visual tone and character of the text can be determined. This lack of guidance led McCarthy and Mothersbaugh (McCarthy and Mothersbaugh, 2002) to conduct research and create a set of principles that link the characteristics of the typeface to the impressions they create. Part of this model has been empirically tested by researching how common typographic characteristics can affect the perception of an advertisement. Although this study does not link the effect of the typeface on conviction (e.g. attitude towards a brand), it in itself indicates that typography has a significant impact on the ability of consumers to read the advertising copy/advertisement. Designers support the idea that typefaces have expressive personalities, suggesting that even a typeface destined to be free from historical and cultural associations cannot be completely free from rhetorical influence (Kinros, 1985; Nedeljković, 2012). Initial research, research by Henderson (Henderson et al, 2004), Childers (Childers, and Jass 2002), Doyle and Bottomley (Doyle and Bottomley, 2006) show that typeface design affects the perception of advertising brands, affects the readability and memory of advertisements, creates strategically important impressions, affects the suitability of the typeface for different products and therefore there is an assumption that it can affect the financial results of the company. Design practitioners had different opinions about the personalities of the typeface. They believe that each typeface has a special personality (Shushan and Wright, 1994; Kostelnick and Roberts, 1998; Brumberger, 2003a), can convey and evoke different feelings and moods, communicate with a certain attitude and adjust the visual tone (Strizver, 2001; Parker and Parker, 1997). These conclusions were further deepened by research where the comparison was made based on the characteristics specific to the typeface. Kastl and Child (Kastl and Child, 1968) concluded 
that there is a connection between certain typeface characters and certain typeface-specific characteristics. While Davis and Smith (Davis and Smith, 1933), striving to find out how specific characteristics affect the impression, concluded that the most extreme differences in size, width and contrast leave the greatest impression on the observer. Exploring the differences between serif and sansserif typefaces, Benson (Benson, 1985) concluded that sans-serif typefaces were cleaner and had a more modern look than sans-serif, while Kostelnick and Roberts (Kostelnick and Roberts, 1998) concluded that sans-serif typefaces were more technical than serif, because of the clean, machine-like looks of modernism. Parker (Parker and Parker 1997) further deepened the research according to the shape of the serif, concluding that rounded, round serifs are more friendly, while the square, slab serifs are more businesslike, i.e. more official. By analyzing the very form of the typeface, it was concluded that thinner strokes are more delicate, gentle, feminine, while thicker strokes are strong, aggressive and masculine (White, 1988; Baylis, 1955), and that smoother flowing strokes with longer ascending and descending strokes give a childlike and friendly atmosphere (Sassoon, 1993). None of the authors mentioned above explains why these typefaces convey these impressions (Mackiewicz and Moeller, 2004). A lot of previous research has been initiated by researchers who do not have academic experience related to typeface design and thus do not have an adequate and professional understanding of typography. This can affect the very setting of the research and its analysis, because due to the lack of understanding of the differences in the formal attributes of each typeface, from weight, skeleton, to the shape and style of the serif.

Recent research (Dyson, 2011; Beier and Larson, 2010; Morris et al, 2002), freed from the technical limitations of the pre-digital design era, can design and control (un)desired variables on a test stimulus, specific to given research.

\section{RESEARCH PROBLEM AND RESEARCH QUESTION}

Based on the review of existing research that attempts to quantify the psychological effects of typographic design, it can be concluded that most research on typography has dealt only with legibility. Typography, in itself, was not considered a semiotic model. In Thames and Hudson's Handbook of Typography, first published in 1980, McLean (Van Leeuwen, 2006) says that "to a very limited extent, typefaces can help express feelings or moods that are consistent with the meaning of the word," but for the most part "typefaces and calligraphic writing are abstract arts"(van Leeuwen, 2006).

Several empirical studies have directly investigated the relationship between typefaces and response. Tantillo, DiLorenzo-Ais and Madison (Rowe, 1982) investigated the influence of different typeface style on selected impressions (e.g., happy/sad and young/old) and found numerous differences between sansserif and serif typefaces while, in a similar study Rowe (Rowe, 1982) found very little difference. Although each of these studies made a worthy initial effort, these studies failed to research the causes of typefaces that have a representative range of design features. This research has also neglected a whole range of responses.

Certain characteristics specific to typeface design leave different impressions on observers, but here a research problem arises in which we cannot see which specific or universal characteristics of typeface affect our perception of the typeface personality itself, and linking it to certain personality attributes. A common problem that has arisen in previous research is that typefaces vary in many variables, and at the same time differ in width, weight, contrast, and skeleton. Therefore, it is difficult to determine specifically which universal and specific characteristics of the typeface affect the very impression of the typeface.

Based on this isolated research problem, we can form a research question:

- $\quad \mathrm{RQ}$ : To what extent and in what way the typeface weight as a factor affects the personality of the typeface?

Guided by this question, we start from two hypotheses:

- $\quad \mathrm{H} 1$ : Based on the estimates of the typeface personality attributes, it is possible to single out the specific typeface personality attributes that are related to the weight variable of the typeface.

- $\quad \mathrm{H} 2$ : Based on the weight variable of the typeface, it is possible to single out specific typeface personality attributes that describe the best and the worst specific typeface weight. 


\section{METHOD}

For the purposes of the research, stimuli were designed with the help of which hypotheses were tested in an experiment with the subjects.

\subsection{Subjects}

Participants in this research are a group of 60 students from the Faculty of Technical Sciences. In preliminary research (Žarko and Nedeljković, 2014), it was noted that there are no significant differences in assessments of the typeface personality attributes between male and female subjects, regardless of whether they are of graphic profession or laymen, so these factors were neglected in this study.

\subsection{Stimuli}

A string of letters, 'Hafonurmtgesbiv', was formed for testing purposes. This string was taken in order not to make any form of suggestion related to the content of the text, while the selection of letters that make up the string contains all the important forms of letters, by which typefaces can be recognized and differentiated.

\subsubsection{Stimulus design}

A prototype of a string of letters is formed, which varies only according to the typeface weight. In this way, one specific characteristic is isolated and its influence on perception will be monitored.

This prototype is defined according to Adrian Frutiger's model (Frutiger, 1998; Frutiger, 2009) of the "common skeleton", which represents a neutral letter shape because its properties are identical to the properties of the fonts used. Frutiger used eight popular fonts (Garamond, Baskerville, Bodoni, Excelsior, Times, Palatino, Optima and Helvetica) in his model. The "common skeleton" was obtained as a crosssection of all the layered fonts used.

The prototype, for the purposes of this research, was formed from Lineal (linéales) typefaces, according to the Vox - Atyp/ classification, where Franklin Gothic was taken as the representative of the Grotesque group; Helvetica was taken for Neo-Grotesque; Futura was taken for Geometry and Gill Sans was taken as Humanistic (Figure 1).

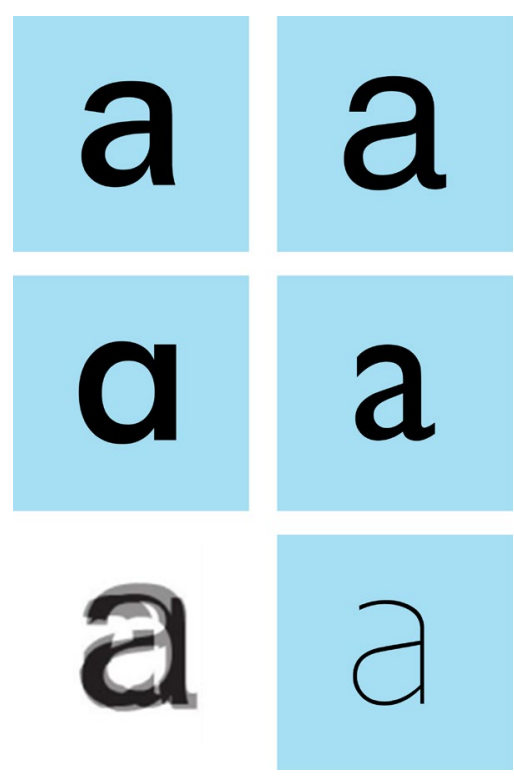

Figure 1: Designing a stimulus based on Frutiger's "common skeleton" model

This string was designed in the program FontLab Studio 5.1 using the option Multiply Master for 10 different typeface weights that follow Panose classification and go in the following order: Ultra Light, Thin, Light, Book, Regular, Medium, Bold, Heavy, Black and Extra Black (Figure 2). 


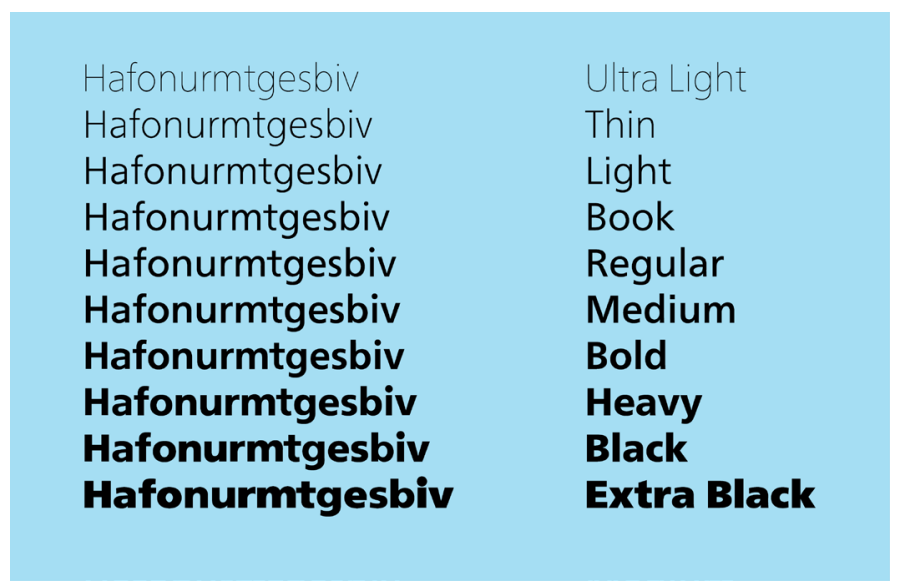

Figure 2: Stimulus model created for 10 different typeface weights

Panose is a classification system for visual attributes of type only. It was originally developed by Benjamin Bauermeister. The premise of Panose is that, by determining the characteristics of the font according to this system, we can identify the closest font match in the group.

The families of the typeface are described with two main characteristics: the manuscript type and the genre type. There are four main categories of manuscript type: Latin, Kanji, Hebrew, and "etc.", which encompasses all fonts that are not included in the first three categories. Genres further classify fonts within the manuscript type. Latin fonts are subclassified as "text", "decorative", "handwritten" or "symbols".

The Latin category represents most Western fonts. Within this family, the font is described using ten digits. The first specifies a subcategory (text, decorative, handwritten, or symbol), and the remaining nine digits indicate a number of additional attributes. For each of these attributes, a number is used to describe a feature. They are predefined in the Panose system. For example, there are fifteen options for a serif attribute. Some of them are: 0 - any, 1 - does not match, 2 - round (cove), 3 - blunt round, 4 rectangular round, 5 - blunt rectangular round. This similar division applies to all nine features. There are thirteen options for the character brightness attribute. 0 - any, 1 - do not match, 2 -Very Light, 3 - Light, 4 - Thin, 5 - Book, 6 - Medium, 7 - Demi, 8 - Bold, 9 - Heavy, 10 - Black n 11 - Extra Black.

In order to create a functional stimulus model for ten typeface weights, it was necessary to make additional perceptual adjustments, however, these methods do not represent a scientific principle in which all character strokes change according to the same mathematical formula.

In order to maintain the same level of contrast of strokes that is predefined in the skeleton, all strokes must have the same weight. However, when weight is added in this way, certain letters, such as "e", " $a$ " and "s", will be congested and closed in the interior. Accordingly, the added weight may not be the same for all letters. The next problem that arises is related to horizontal and vertical strokes. To make vertical and horizontal strokes look equal in weight, horizontal strokes should be lighter in weight than vertical strokes. This problem is best seen with the letter " $H "$ ". This is a consequence of our perceptual understanding, where we do not see all shapes and forms in their mathematically correct dimensions (Gates, 1969; Beier, 2013).

\subsubsection{Binary code matrix analysis}

The validity of the designed stimuli was further tested through digital image analysis in digital image processing software, ImageJ. Ratio and range validation were measured using mean grey value and structural similarity analysis - SSIM (The Structural Similarity Index).

\subsubsection{Mean grey value}

The analysis of the mutual relationship between the ten different typeface weights was done by determining their mean grey value. Mean grey value analysis is often used during the analysis of raster digital images. The advantage of this procedure is image segmentation, as well as limiting the influence of filters in the very process of image processing. The mean grey value is defined as the sum of the intensity values of all pixels present in the image divided by the total number of pixels. The formula used to calculate the value of the mean grey is (Nedeljković et al, 2012): 


$$
P_{\text {avg }}=\frac{1}{M \times N} \sum_{i=1}^{M} \sum_{j=1}^{N} I_{m a t}(i, j)
$$

where $\mathrm{M}$ and $\mathrm{N}$ represent the number of rows and columns digitized, Imat $(i, j)$ are mean grey values on position $(i, j)$.

Ten measuring samples with the dimensions of $2262 \times 394$ pixels with the presented string of letters 'Hafonurmtgesbiv' were created. The results of mean grey measurements are given in Table 1.

Table 1: Mean grey value

\begin{tabular}{|c|c|c|c|}
\hline & Mean grey value & \% of decrease of the mean grey value & Std. Dev \\
\hline Ultra Light & 249,70 & $0 \%$ & 34,86 \\
\hline Thin & 243,85 & $-2 \%$ & 51,13 \\
\hline Light & 239,47 & $-2 \%$ & 60,12 \\
\hline Book & 236,07 & $-1 \%$ & 65,96 \\
\hline Regular & 232,96 & $-1 \%$ & 70,88 \\
\hline Medium & 229,53 & $-1 \%$ & 75,76 \\
\hline Bold & 225,80 & $-2 \%$ & 80,51 \\
\hline Heavy & 221,10 & $-2 \%$ & 85,93 \\
\hline Black & 216,61 & $-2 \%$ & 90,59 \\
\hline Extra Black & 209,82 & $-3 \%$ & 96,76 \\
\hline
\end{tabular}

\subsubsection{Structural similarity analysis SSIM}

After the analysis of the mean grey value, using the same measurement samples, the analysis of structural similarity (SSIM) was conducted. SSIM measures the similarity between two images using three essential factors: brightness, contrast, and structure. The SSIM index is calculated through the formula (Wang et al., 2003):

$\operatorname{SSIM}(x, y)=\frac{\left(2 \mu_{x} \mu_{y}+c_{1}\right)\left(2 \sigma_{x y}+c_{2}\right)}{\left(\mu_{x}^{2}+\mu_{y}^{2}+c_{1}\right)\left(\sigma_{x}^{2}+\sigma_{y}^{2}+c_{2}\right)}$

where $x, y$ are the images on which we examine similarity, $\mu x$ - the average value of $x ; \mu y$ - the average value of $\mathrm{y} ; \sigma_{x}^{2}$ - variance of $\mathrm{x} ; \sigma_{y}^{2}$ - variance of $\mathrm{y} ; \sigma_{\mathrm{xy}}$ - covariance of $\mathrm{x}$ and $\mathrm{y} ; \mathrm{c}_{1}$ and $\mathrm{c}_{2}-$ are variables that serve to stabilize the negative denominator in the fraction.

The $\operatorname{SSIM}$ index satisfies the symmetry condition $\operatorname{SSIM}(x, y)=\operatorname{SSIM}(y, x)$. The $\operatorname{SSIM}$ index result is represented as a decimal value from -1 to 1 . A value of 1 is only possible in the case of two identical data sets. Ten measuring samples of the letter string 'Hafonurmtgesbiv' with different typeface weight were compared with each other. The results obtained in this way show how different a specific typeface weight is compared to the others (Table 2).

Table 2: SSIM index value

\begin{tabular}{|c|c|c|c|c|c|c|c|c|c|c|}
\hline & Ultra Light & Thin & Light & Book & Regular & Medium & Bold & Heavy & Black & $\begin{array}{c}\text { Extra } \\
\text { Black }\end{array}$ \\
\hline Ultra Light & 1 & $/$ & $/$ & $/$ & $/$ & $/$ & $/$ & $/$ & $/$ & $/$ \\
\hline Thin & 0,956 & 1 & $/$ & $/$ & $/$ & $/$ & $/$ & $/$ & $/$ & $/$ \\
\hline Light & 0,953 & 0,955 & 1 & $/$ & $/$ & $/$ & $/$ & $/$ & $/$ & $/$ \\
\hline Book & 0,952 & 0,952 & 0,950 & 1 & $/$ & $/$ & $/$ & $/$ & $/$ & $/$ \\
\hline Regular & 0,950 & 0,950 & 0,950 & 0,952 & 1 & $/$ & $/$ & $/$ & $/$ & $/$ \\
\hline Medium & 0,950 & 0,950 & 0,949 & 0,947 & 0,948 & 1 & $/$ & $/$ & $/$ & $/$ \\
\hline Bold & 0,948 & 0,948 & 0,947 & 0.946 & 0,947 & 0,947 & 1 & $/$ & $/$ & $/$ \\
\hline Heavy & 0,943 & 0,943 & 0,944 & 0,945 & 0,945 & 0,947 & 0,949 & 1 & $/$ & $/$ \\
\hline Black & 0,942 & 0,942 & 0,942 & 0,942 & 0,942 & 0,943 & 0,943 & 0,946 & 1 & $/$ \\
\hline Extra Black & 0,935 & 0,935 & 0,936 & 0,936 & 0,936 & 0,937 & 0,940 & 9,40 & 0,941 & 1 \\
\hline
\end{tabular}




\subsection{Test instrument}

20 typeface personality attributes were examined for 10 typeface weights of the string 'Hafonurmtgesbiv'. Typeface personality attributes consist of 20 descriptors collected from previous research (Brumberger, 2003b; Davis and Smith, 1933; Henderson, 2004; Mackiewicz and Moeller, 2004; Nedeljković et al, 2012; Rowe, 1982): Cheap, Cold, Reliable, Dignified, Elegant, Feminine, Formal, Friendly, Attractive, Loud, Masculine, Contemporary, Pretentious, Professional, Relaxed, Academic, Serious, Technical, Direct and Warm. They were rated on the Likert scale ranging from 1 to 7 . Subjects were not given any explanation or description of the research method, as such explanations could influence subjects' decisions to respond in a certain way.

For the purposes of the examination, a booklet was formed consisting of 10 wired pages containing the string of letters 'Hafonurmtgesbiv'. The pages were randomly shuffled so that the continuity of the typeface weight change is not obvious.

\section{RESULT ANALYSIS AND DISCUSSION}

Data collected by the conducted experiment with the subjects were analyzed using the program SPSS 20.

\subsection{Results of research with subjects}

The impact of the typeface personality attribute variable was analyzed using arithmetic means as the mean values of the subjects' estimates for all presented descriptors. Based on the results, certain rules in the perception of the typeface can be noted. As the typeface weight increases, the feeling of Femininity and Elegance is decreasing (Figure 3a). Contrary to the typeface personality attribute Feminine, it can be noted that with the increase in the typeface weight, the influence of Loudness and Masculinity typeface personality attribute increases (Figure $3 b$ ).

A similar trend was noted in typeface personality attributes Professional, Academic, Serious and Technical (Figure 4), while with the typeface personality attributes Direct and Pretentious no trend was noted (Figure 5).

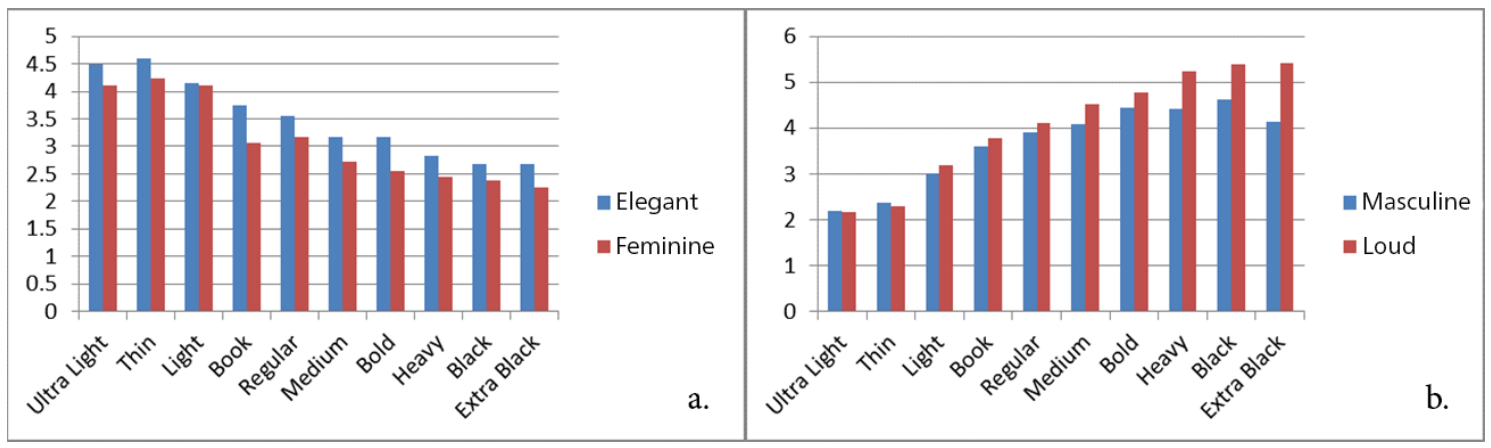

Figure 3: a. Comparison of results for the attributes Feminine and Elegant;

b. Comparison of results for the attributes Masculine and Loud

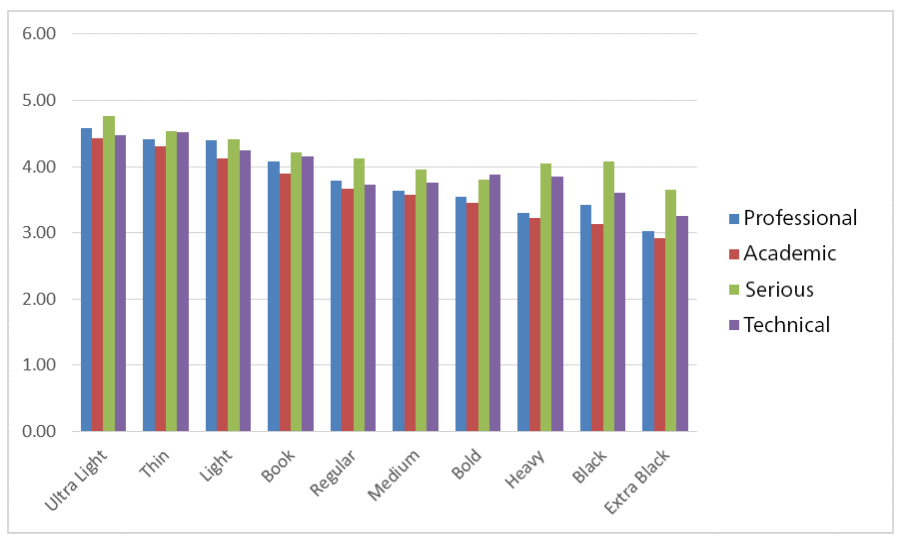

Figure 4: Comparison of results for the attributes Professional, Academic, Serious and Technical 


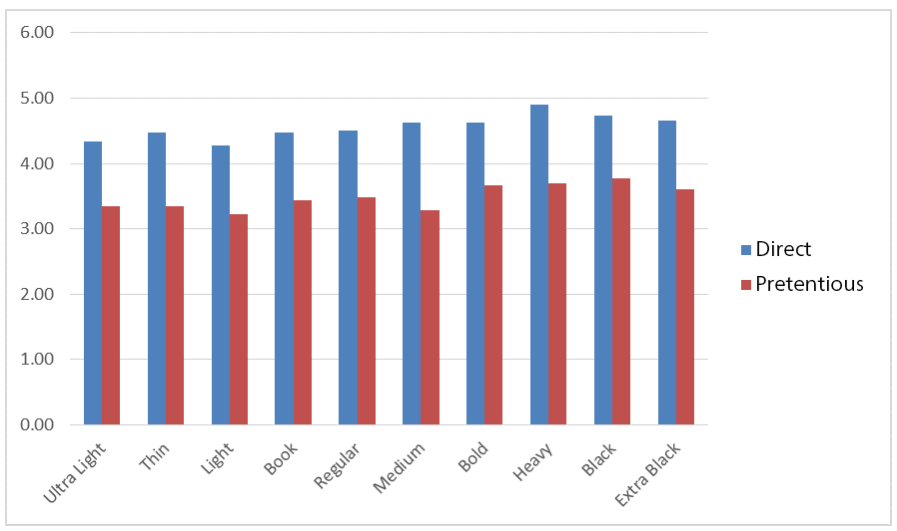

Figure 5: Comparison of results for the attributes Direct and Pretentious

\subsubsection{Factor analysis of the main components on the typeface weights}

To examine the possibility of a grouping of the weights and possible identification of common factors, a factor analysis of the main components with Varimax rotation and Kaiser normalization was performed. When Factor includes only the sums on the typeface weights, three factors stand out that describe $71 \%$ of the variance. The first factor consists of the following weights: Medium, Bold, Heavy, Black and Extra Black. The second factor consists of the following weights: Thin, Light, Book and Regular. The third factor consists of the following weight: Ultra Light. The first factor is best defined with the typeface personality attributes Loud, Masculine and Direct. It is least defined with typeface personality attributes Feminine, Elegant and Academic. The second factor is best defined with typeface personality attributes Elegant, Academic, Professional and Serious, and the least with typeface personality attribute cheap. The third factor is best defined with typeface personality attributes Professional, Formal and Elegant, and the least by the typeface personality attributes loud and masculine.

Since the distributions of subjects' responses do not follow statistical normality, a nonparametric KruskalWallis Test was used to compare several groups. Looking at the statistically significant differences, they are shown on several typeface personality attributes. The typeface personality attributes where there is statistically significant difference are Dignified, Elegant, Feminine, Formal, Friendly, Attractive, Loud, Masculine, Professional, Academic, Serious, Technical and Warm.

\subsubsection{Factor analysis of the main components on the typeface personality attributes}

Factor analysis was performed on the selected typeface personality attributes. Prior to the analysis, the possibility of applying factor analysis (KMO test and Bartlett test) was tested. According to Scree Plot (Figure 6), it is possible to single out one most important factor, or three significant ones. The first factor describes $37.12 \%$ of the variance, while all three factors together describe $66 \%$ of the variance.

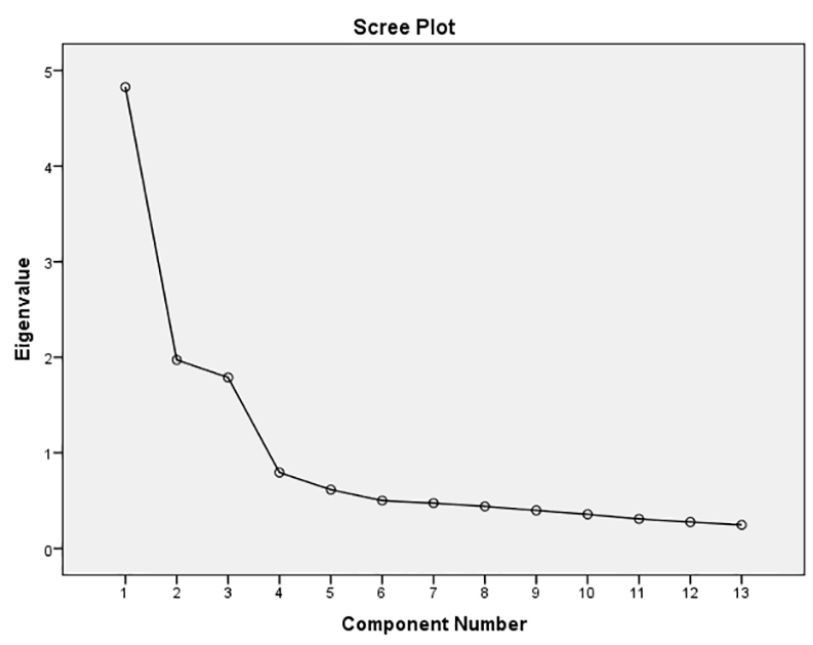

Figure 6: Scree plot of the factor analysis 
When only one factor is singled out, it is best described by the typeface personality attributes: Professional, Elegant, Formal, Dignified, Academic and Attractive. On the other hand, this factor excludes the typeface personality attributes such as Warm, Loud and Masculine. The excluded typeface personality attributes contribute very little or nothing to the factor.

When three factors are analyzed, the first factor includes the typeface personality attributes Professional, Serious, Academic, Formal, Technical and Dignified. The second factor includes the typeface personality attributes Attractive, Friendly, Elegant, Feminine and Warm. The third factor is described by the typeface personality attributes Masculine and Loud. These factors, with the typeface personality attributes that define them, follow the changing trend of the typeface weight. The most prevalent is certainly the first factor, but all three factors are statistically significant. To confirm that the isolated factors are correlated, the Promax rotation was used. Promax rotation analysis confirmed that the first and the second factors correlate statistically significantly in the positive direction $(r=0.456)$, while the third factor correlates slightly and in a negative direction.

To observe which of these factors best describes which group of typeface weights, a one-way analysis of variance was performed. The independent variable was the typeface, while the dependent variables were isolated factors. According to the applied statistical analysis, it can be said that the typeface weights differ statistically significant from each other according to each of the factors. This is shown by the size of $f$ test and significance. The Post hoc test (Sheffe's) was used to show on which factors are statistically significant differences. It is necessary to emphasize that Sheffe's post hoc test is stricter in its criteria compared to the applied one-way analysis of variance.

The first factor divides the typeface weights into four groups, within which there are no statistically significant differences, and yet it exists with the next group. The noticeable trend is that the lighter the typeface weight is, the more pronounced this factor is, while the darker typeface weight is negative. No statistically significant difference was noted for the second factor. But it is noticeable how the typeface weights are arranged according to this factor. From weights which describe it in a negative direction (Extra Black), to weights which have a highly positive trend towards it (Light). The third factor divides the typeface weights into five groups, within which there are no statistically significant differences, yet it exists with the next group. It is noticeable that the lighter the typeface weight, the more negative is the factor, while the darker typeface weight is the most pronounced is the factor.

Based on these results, it is noticeable that the first factor, which includes the typeface personality attributes Professional, Serious, Academic, Formal, Technical and Dignified, best describes the weights Ultra Light, Thin, Light and Book. The second factor, which includes the typeface personality attributes Attractive, Friendly, Elegant, Feminine and Warm, best describes the weights Ultra Light, Thin, Light, Book and Regular. The third factor, which includes the typeface personality attributes Male and Loud, best describes the weights Extra Black, Black, Heavy, Bold and Medium. It can be noted that in all three groups of factors, with the increase in the typeface weight also increases the attitude towards the given typeface personality attribute. In the case of the first factor, which includes the typeface personality attributes Professional, Serious, Academic, Formal, Technical and Dignified, these attributes are most pronounced in the weight Ultra Light and their expression decreases linearly with the increase of the weight, where it is least pronounced in the weight Extra Black. The same case is with the second factor, which includes the typeface personality attributes Attractive, Friendly, Elegant, Feminine and Warm, these are most pronounced in lighter weights and their expression decreases linearly with the increase of the weight. In case of the third factor, which includes the typeface personality attributes Masculine and Loud, the expression of these attributes increases linearly with the increase of weight, i.e. they are most pronounced in the weight Extra Black and least in the weight Ultra Light.

\section{CONCLUSION}

Researching the topic of the typeface personality, it is noticeable that the previous research on this topic are incomplete. They do not provide answers to all questions and do not take into account all possible aspects that could affect the impressions that the typefaces leave. This research was conducted with the goal to make a functional classification of the typefaces that connects all aspects of the typeface personality attributes and visual rhetoric in such a way that will be functional, understandable and accessible to the public regardless of the level of education. The results showed that the participants consistently attribute certain typeface personality attributes to certain typeface weights.

The results also confirmed the assumptions that there are significant differences between typeface personality attributes. Factor analysis of the main components, which includes only the typeface weights, 
shows that typeface weights were grouped into three factors that differentiate for each typeface weight (Figure 7). The results showed which typeface personality attributes describe each group of typeface weights. It is noticeable that the typeface weights were grouped according to the weights between which are lower percentage contrast, which matches the results of the mean grey values (Table 1 ).

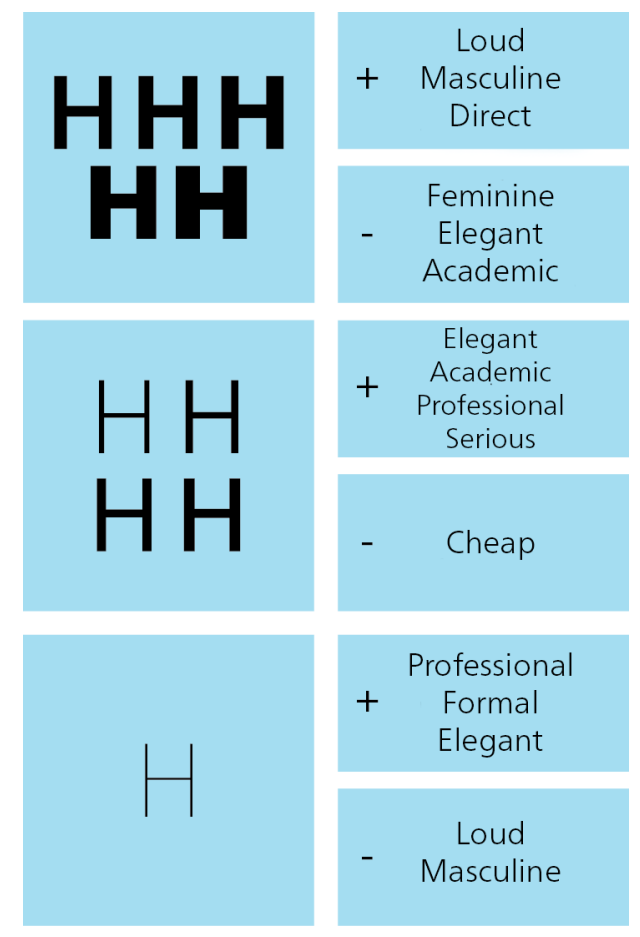

Figure 7: Results of factor analysis of the main components on the typeface weights

Besides, based on the results of factor analysis of the main components on the typeface personality attributes, it is noted that they form three groups of factors in which increasing/decreasing the typeface weight affects the increase/decrease of attitude towards the typeface personality attributes. The first factor, which consists of the typeface personality attributes Professional, Serious, Academic, Formal, Technical and Dignified, are most pronounced in the weight Ultra Light and their expression decreases linearly with increase of the weight, and it is least pronounced in the weight Extra Black. The same case is with the second factor, which includes the typeface personality attributes Attractive, Friendly, Elegant, Feminine and Warm, these attributes are most pronounced in lighter weights and their expression decreases linearly with increasing of weight. The third factor, which includes the typeface personality attribute Masculine and Loud, where the expression of these attributes increases linearly with the increase of weight, i.e. they are most pronounced in the weight Extra Black and least pronounced in the weight Ultra Light.

Based on results, we can conclude that the typeface weight is an important factor, which should be paid attention to when choosing a typeface. Proper choice of weight affects the tone and attitude towards the text itself.

A new research question arises here, to what extent and in what way does the typeface weight as a factor can affect the personality in serif letters? For this kind of research, it will be necessary to create a new set of typeface weights with serifs as an additional feature.

\section{ACKNOWLEDGMENTS}

I would like to express my gratitude and appreciation for Uroš Nedeljković whose guidance, support and encouragement have been invaluable throughout this research.

Also, I would like to thank my mother Dragica, sister Ana and my love Vladimir for their wise counsel and sympathetic ear. You are always there for me. I could not have completed this research without the support of my friends, Mitar and Borjana, who provided stimulating discussions as well as happy distractions to rest my mind outside of my research. 
Finally, many thanks to all participants that took part in the study and enabled this research to be possible.

\section{REFERENCES}

[1] Aristotele, Roberts, W. R., Bywater, I. Solmsen, F.: "Rhetoric", (Modern Library, New York, 1954.)

[2] Baylis, C.: "Trends in typefaces", Printer's ink 252 (5), 44-46, 1955.

[3] Beier, S., Larson, K.: "Design Improvements for Frequently Misrecognized Letters", Information Design Journal 18 (2), 118-137, 2010.

[4] Beier, S.: "Legibility Investigations: Controlling Typeface Variables", Praxis and Poetics: Research Through Design 2013 Conference Proceedings, (Northumbria University: New Castle, United Kingdom, 2013), pages 92-95.

[5] Benson, P.: "Writing visually: Design considerations in technical publications", Technical communication 32 (4), 35-39, 1985. doi: 10.1177/1050651994008001004

[6] Brumberger, E.: "The rhetoric of typography: The persona of typeface and text", Technical Communication 50 (2), 206-223, 2003.

[7] Brumberger, E.: "The rhetoric of typography: The awareness and impact of typeface appropriateness", Technical Communication 50 (2), 224-231, 2003.

[8] Childers T., Jass J.: "All Dressed Up with Something to Say: Effects of Typeface Semantic Associations on Brand Perceptions and Consumer Memory", Journal of Consumer Psychology 12 (2), 93-106, 2002. doi: 10.1207/S15327663JCP1202_03

[9] Davis, R. C., Smith, H. J.: "Determinants of Feeling Tone in Type Faces", Journal of Applied Psychology 17, 742-764, 1933. doi: 10.1037/h0074491

[10] Dyson, M. C.: “Do Designers Show Categorical Perception of Typefaces?", Visible Language 45 (3), 193-220, 2011

[11] Doyle J., Bottomley P.: "Dressed for the Occasion: Font-Product Congruity in the Perception of Logotype", Journal of consumer psychology 16 (2), 112-123, 2006. doi: 10.1207/s15327663jcp1602_2

[12] Frutiger, A.: "Signs and Symbols: Their Design and Meaning", (Ebury Press, London, 1998.)

[13] Frutiger A.: "Typefaces: The Complete Works", (Springer Verlag, New York, 2009.), ISBN $3764385812,9783764385811$.

[14] Gates, D.: "Lettering for Reproduction", (Watson-Guptill Publication, New York, 1969.)

[15] Henderson, P.W., Giese, J.L., Cote, J.A.: "Impression management using typeface design", Journal of Marketing 68 (4), 60-72, 2004. doi: 10.1509/jmkg.68.4.60.42736

[16] Kastl, A. J., Child, I. L.: "Emotional meaning of four typographical variables.", Journal of Applied Psychology 52 (6), 440, 1968. doi: 10.1037/h0026506

[17] Kinross, R.: "The rhetoric of neutrality", Design Issues 2 (2), 18-30, 1985. doi: 10.2307/1511415

[18] Kostelnick, C., Roberts, D.: "Designing visual language: Strategies for professional communicators", (Allyn and Bacon, Needham Heights, 1998.)

[19] Mackiewicz, J., Moeller, R.: "Why people perceive typefaces to have different personalities", Proceedings: Communication Frontiers: IPCC 2004, (IEEE: Minneapolis, USA, 2004), pages 304-313.

[20] McLean R.: "Manual of Typography", (Thames \& Hudson Ltd, London, 1980), ISBN-10: 0500670226.

[21] McCarthy, M., Mothersbaugh, D.: "Effects of typographic factors in advertising-based persuasion: A general model and initial empirical tests", Psychology \& Marketing 19 (7-8), 663-691, 2002. doi: 10.1002/mar.10030

[22] Morris, R. A., Aquilante, K., Yager, D., Bigelow, C.: "P-13: Serifs Slow RSVP Reading at Very Small Sizes, but Don't Matter at Larger Sizes", SID Symposium Digest of Technical Papers 33 (1), (Blackwell Publishing Ltd: Oxford, United Kingdom, 2002), pages 244-247.

[23] Nedeljković U., Novaković D., Pinćjer I.: "Detecting Universal Structure and Effects of Typefaces", Technical Gazette 19 (4), 709-715, 2012. doi: 10.17559/TV-20150831131738

[24] Parker, C. R., Parker R.: "Looking good in print", (Ventana Communications Group Inc, Research Triangle Park NC, 1997.)

[25] Rowe, Camille L.: "The connotative dimensions of selected display typefaces", Information design journal 3 (1), 30-37, 1982. doi: 10.1075/idj.3.1.03row

[26] Sassoon, R.: "Through the eyes of a child - Perception and type design" In Computers and typography, (IntellectBooks, Oxford UK, 1993.), pages 178-201.

[27] Shushan, R., Wright, D.: "Desktop publishing by design", (Microsoft Press, Redmond WA, 1994.) 
[28] Strizver, I.: "Type Rules: The designer's guide to professional typography", (John Wiley \& Sons, Hoboken NJ, 2001.)

[29] Trummel, P.: "Rhetoric+typography: creative interaction in modern communication", IEEE transactions on professional communication 31 (3), 124-129, 1988.

[30] Van Leeuwen, T.: "Towards a semiotics of typography", Information design journal 14 (2), 139-155, 2006. doi: 10.1075/idj.14.2.06lee

[31] Wang, Z., Simoncelli, E. P., Bovik, A. C.: "Multiscale structural similarity for image quality assessment", The Thrity-Seventh Asilomar Conference on Signals, Systems \& Computers 2003, (IEEE: Pacific Grove CA, USA, 2003)

[32] White, J.: "Graphic design for the electronic age: The manual for traditional and desktop publishing", (NYT: Watson-Guptill Publications and Xerox Press, New York, 1988.)

[33] Žarko, J., Nedeljković, U.: “Efekti svetline lika na procenu atributa ličnosti tipografskog pisma”, Zbornik radova Fakulteta tehničkih nauka 29 (12), 2577-2580, 2014.

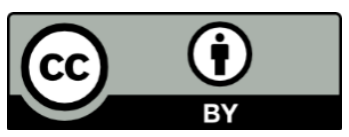

(C) 2020 Authors. Published by the University of Novi Sad, Faculty of Technical Sciences, Department of Graphic Engineering and Design. This article is an open access article distributed under the terms and conditions of the Creative Commons Attribution license 3.0 Serbia (http://creativecommons.org/licenses/by/3.0/rs/). 